\title{
ON THE ISOMONODROMIC TAU-FUNCTION FOR THE HURWITZ SPACES OF BRANCHED COVERINGS OF GENUS ZERO AND ONE
}

\author{
Alexey Kokotov, Ian A. B. Strachan
}

\begin{abstract}
The isomonodromic tau-function for the Hurwitz spaces of branched coverings of genus zero and one are constructed explicitly. Such spaces may be equipped with the structure of a Frobenius manifold and this introduces a flat coordinate system on the manifold. The isomonodromic tau-function, and in particular the associated $G$-function, are rewritten in these coordinates and an interpretation in terms of the caustics (where the multiplication is not semisimple) is given.
\end{abstract}

\section{Introduction}

The equations which define the monodromy preserving deformations of the Fuchsian system of differential equations

$$
\frac{d \Psi}{d \lambda}+\sum_{k=1}^{M} \frac{A_{k}\left(\lambda_{1}, \ldots, \lambda_{M}\right)}{\lambda-\lambda_{k}} \Psi=0
$$

were derived by Schlesinger in 1912 and since then, particularly after the seminal work of Jimbo and Miwa, they have been the source of inspiration for many mathematicians; the equations having many beautiful analytic, algebraic and geometric properties. Naturally associated to such a system is a closed 1-form $\omega$ from which one may define a function $\tau$ via $\omega=d \log \tau$. It is this function that is the central object of study in this paper. Such $\tau$-functions have appeared in recent work on topics such as the computation of determinants of Laplace operators on Riemann surfaces and the calculation of the asymptotic expansions in the theory of random matrices [4].

The application of Schlesinger's equations that will be considered in this paper comes from the theory of Frobenius manifolds. These were introduced by Dubrovin [1] as a geometric way to describe the associativity equations that arise in topological quantum field theory (the so-called Witten-Dijkgraaf-VerlindeVerlinde equations). For semisimple Frobenius manifolds one may reformulate the manifold's defining relations as a reduction of Schlesinger's equations. In this specific context these reduced equations have two interesting properties:

- the appearance of non-Fuchsian singularities, 'wild' monodromies and Stokes matrices;

Received by the editors February 27, 2004. 
- the existence of an alternative, distinguished, coordinate system - the socalled flat coordinates $\left\{t^{i}\right\}$.

The associated $\tau$-function for such systems will be written as $\tau_{I}$ and called the isomonodromic $\tau$ function ${ }^{1}$. This function also plays a major role in the formula, conjectured by Givental [7] and proved by Dubrovin and Zhang [2] for the $G$-function of a semisimple Frobenius manifold

$$
G=\log \left\{\frac{\tau_{I}}{J^{\frac{1}{24}}}\right\}
$$

where

$$
J=\operatorname{det}\left(\frac{\partial\left(t^{1}, \ldots, t^{n}\right)}{\partial\left(\lambda^{1}, \ldots, \lambda^{n}\right)}\right)
$$

is the Jacobian of the tranformation between the flat coordinates $\left\{t^{i}\right\}$ and the canonical coordinates $\left\{\lambda^{i}\right\}$. This function plays a number of roles in the theory of Frobenius manifolds all related with the construction of first order (or genus 1) objects from zeroth order (or genus 0) data. For example, it appears in the genus 1-term in the free energy of the related topological quantum field theory, in the generating functions for genus 1-Gromov-Witten invariants, in first order terms in the expansion of oscillatory integrals and in first order deformations of biHamiltonian structures.

A particular class of Frobenius manifolds may be constructed on Hurwitz spaces - moduli spaces of meromorphic functions on Riemann surfaces. (see [1] and section 2). In this paper the $\tau_{I}$ and $G$-functions for genus 0 and 1 Hurwitz spaces are studied. In section 2 these functions will be constructed in terms of critical data on the Riemann surface. In section 3 the formulae will be reevaluated in terms of flat-coordinates, and this enables certain global properties of these functions to be studied.

It will turn out that the non-semisimple part of the manifold plays a pivotal role. By definition, a massive Frobenius manifold $M$ has a semisimple multiplication on the tangent space at generic points of $M$. The set of points where the multiplication is not semisimple is known as the caustic, and will be denoted $\mathcal{K}$. This is an analytic hypersurface in $M$, which may consist of a number of components (possibly highly singular),

$$
\mathcal{K}=\bigcup_{i=1}^{\# \mathcal{K}_{i}} \mathcal{K}_{i}
$$

This forms part of the bifurcation diagram $\mathcal{B}=\left\{\lambda: \lambda^{i}-\lambda^{j}=0, i \neq j\right\}$ and in general $\mathcal{B} \cong \mathcal{K} \cup \mathcal{M}$ where $\mathcal{M}$ is the so-called Maxwell strata. The components of the caustic are given in terms of quasi-homogeneous irreducible polynomials $\kappa_{i}$ such that $\kappa_{i}^{-1}(0)=\mathcal{K}_{i}$. For examples, using the ideas developed in [14] one

\footnotetext{
${ }^{1} \mathrm{~A}$ factor of $-1 / 2$ is missing in the definition of the closed form $\omega$ given in [2] ([12]). Here we use the Dubrovin and Zhang formulae and hence the $\tau_{I}$-functions that appear in this paper are related to the 'true' $\tau$-function by the formula $\tau_{I}=\tau^{-\frac{1}{2}}$. This has the effect of interchanging the zero and pole loci of the function.
} 
may show that the isomonodromic $\tau$-function for the Frobenius manifold $\mathbb{C}^{N} / W$ where $W$ is a Coxeter group, is given by

$$
\tau_{I}=\prod_{i=1}^{\# \mathcal{K}_{i}} \kappa_{i}^{-\frac{\left(N_{i}-2\right)^{2}}{16 N_{i}}}
$$

where the $N_{i}$ are certain integers determining the F-manifold structure on the caustics [8]. Such a formula determines certain global properties of the function, such as its zeros and poles and its irreducibility properties. The case $W=A_{N-1}$ (where $\# \mathcal{K}_{i}=1$ and $N_{1}=3$ ) coincides with the particular Hurwitz space $H_{0, N}(N)$.

\section{The isomonodromic tau-function}

In this section we give a short proof of the formulae for the isomonodromic tau-function of semisimple Frobenius manifolds associated to the Hurwitz spaces of branched coverings of genus 0 and 1 . Closely related formulae for the isomonodromic tau-function of the class of Riemann-Hilbert problems solved in [11] were first found in [9] without reference to any associated Frobenius manifold. The connections between the formulae in [9] and Frobenius manifolds were conjectured in [14] and finally established in [10]. These formulae were proved indirectly; the method was based on a detailed study of the behaviour of some regularized Dirichlet integrals under deformations of the branched coverings. The introduction of Dirichlet integrals was inspired by the paper [17] on accessory parameters which appeared, at least superficially, to deal with a similar question.

Such methods use auxiliary structures not directly connected with the holomorphic geometry of Hurwitz spaces. It was conjectured in [9] that such indirect methods are inappropriate and can be replaced by a direct one which should use only holomorphic geometry. Here we present such a proof.

\subsection{Preliminaries.}

2.1.1. Hurwitz spaces and Frobenius manifolds. Frobenius manifolds related to Hurwitz spaces were introduced by Dubrovin (see [1], chapter 5). It will be assumed that the reader is familiar with this construction. Here we recall some basic facts and definitions in order to fix notation.

Let $H_{g, N}\left(k_{1}, \ldots, k_{l}\right)$ be the Hurwitz space ${ }^{2}$ of equivalence classes $\left[p: \mathcal{L} \rightarrow \mathbb{P}^{1}\right]$ of $N$-fold branched coverings $p: \mathcal{L} \rightarrow \mathbb{P}^{1}$, where $\mathcal{L}$ is a compact Riemann surface of genus $g$ and the holomorphic map $p$ of degree $N$ is subject to the following conditions:

- it has $M$ simple ramification points $P_{1}, \ldots, P_{M} \in \mathcal{L}$ with distinct finite images $\lambda_{1}, \ldots, \lambda_{M} \in \mathbb{C} \subset \mathbb{P}^{1}$;

\footnotetext{
${ }^{2}$ Dubrovin uses a slightly different notation. In his notation the Hurwitz space is $H_{g ; k_{1}-1, \ldots, k_{l}-1}$.
} 
- the preimage $p^{-1}(\infty)$ consists of $l$ points: $p^{-1}(\infty)=\left\{\infty_{1}, \ldots, \infty_{l}\right\}$, and the ramification index of the map $p$ at the point $\infty_{j}$ is $k_{j}\left(1 \leq k_{j} \leq N\right)$.

(We define the ramification index at a point as the number of sheets of the covering which are glued together at this point. A point $\infty_{j}$ is a ramification point if and only if $k_{j}>1$. A ramification point is simple if the corresponding ramification index equals 2.) The Riemann-Hurwitz formula implies that the dimension of this space is $M=2 g+l+N-2$. One has also the equality $k_{1}+\cdots+k_{l}=N$. Two branched coverings $p_{1}: \mathcal{L}_{1} \rightarrow \mathbb{P}^{1}$ and $p_{2}: \mathcal{L}_{2} \rightarrow \mathbb{P}^{1}$ are said to be equivalent if there exists a biholomorphic map $f: \mathcal{L}_{1} \rightarrow \mathcal{L}_{2}$ such that $p_{2} f=p_{1}$.

We also introduce the covering $\hat{H}_{g, N}\left(k_{1}, \ldots, k_{l}\right)$ of the space $H_{g, N}\left(k_{1}, \ldots, k_{l}\right)$ consisting of pairs

$$
<\left[p: \mathcal{L} \rightarrow \mathbb{P}^{1}\right] \in H_{g, N}\left(k_{1}, \ldots, k_{l}\right),\left\{a_{\alpha}, b_{\alpha}\right\}_{\alpha=1}^{g}>,
$$

where $\left\{a_{\alpha}, b_{\alpha}\right\}_{\alpha=1}^{g}$ is a canonical basis of cycles on the Riemann surface $\mathcal{L}$. The spaces $\hat{H}_{g, N}\left(k_{1}, \ldots, k_{l}\right)$ and $H_{g, N}\left(k_{1}, \ldots, k_{l}\right)$ are connected complex manifolds and the local coordinates on these manifolds are given by the finite critical values of the map $p$, namely $\lambda_{1}, \ldots, \lambda_{M}$. For $g=0$ the spaces $\hat{H}_{g, N}\left(k_{1}, \ldots, k_{l}\right)$ and $H_{g, N}\left(k_{1}, \ldots, k_{l}\right)$ coincide.

Let $\phi$ be a primary differential (see [1]) on the Riemann surface $\mathcal{L}$. With this one may induce the structure of a semisimple Frobenius manifold $M_{\phi}$ on $\hat{H}_{g, N}\left(k_{1}, \ldots, k_{l}\right)$ by defining: the multiplication law on the tangent bundle $\partial_{\lambda_{m}}$ ○ $\partial_{\lambda_{n}}=\delta_{m n} \partial_{\lambda_{m}}$; the unity $e=\sum_{m=1}^{M} \partial_{\lambda_{m}}$; the Euler field $E=\sum_{m=1}^{M} \lambda_{m} \partial_{\lambda_{m}}$ and the one-form $\Omega_{\phi^{2}}=\sum_{m=1}^{M}\left\{\operatorname{Res}_{P_{m}}\left(\phi^{2} / d \lambda\right)\right\} d \lambda_{m}$, where $\lambda$ is the coordinate on the Riemann surface $\mathcal{L}$ lifted from the base $\mathbb{P}^{1}$.

The invariant metric $\xi(v, w)=\Omega_{\phi^{2}}(v \circ w)$ on the Frobenius manifold is flat and potential. In the coordinates $\lambda_{1}, \ldots, \lambda_{M}$ this metric is diagonal: $\xi=$ $\sum_{m=1}^{M} \xi_{m m}\left(d \lambda_{m}\right)^{2}, \xi_{m m}=\operatorname{Res}_{P_{m}}\left(\phi^{2} / d \lambda\right)$. The rotation coefficients, $\gamma_{m n}(m \neq$ $n)$, of this metric are defined by the equality

$$
\gamma_{m n}=\frac{\partial_{\lambda_{n}} \sqrt{\xi_{m m}}}{\sqrt{\xi_{n n}}}
$$

Since the metric is flat there also exists a further distinguished coordinate system known as the flat coordinates $\left\{t^{i}\right\}$ (defined up to linear transformations) in which the components of the metric are constant. The Jacobian (3) of the transformation between the flat and canonical coordinate systems is given by

$$
J^{2}=\prod_{m=1}^{M} \xi_{m m}=\prod_{m=1}^{M} \operatorname{Res}_{P_{m}}\left(\phi^{2} / d \lambda\right) .
$$

2.1.2. The isomonodromic tau-function of a semisimple Frobenius manifold. Let $M$ be a semisimple Frobenius manifold with canonical coordinates $\lambda_{1}, \ldots, \lambda_{M}$ and invariant metric $\xi$ having rotation coefficients $\gamma_{m n}$. Let $\mathcal{U}=\operatorname{diag}\left(\lambda_{1}, \ldots, \lambda_{M}\right)$, $\Gamma=\left\|\gamma_{m n}\right\|_{m, n=1, \ldots, M ; m \neq n}$, and

$$
V=[\Gamma, \mathcal{U}]
$$


The isomonodromic tau-function $\tau_{I}$ of the semisimple Frobenius manifold $M$ is defined by the system of (compatible) equations

$$
\frac{\partial \log \tau_{I}}{\partial \lambda_{m}}=H_{m}, \quad m=1, \ldots, M,
$$

where the quadratic Hamiltonians $H_{m}$ are defined by

$$
H_{m}=\frac{1}{2} \sum_{n \neq m ; 1 \leq n \leq M} \frac{V_{n m}^{2}}{\lambda_{m}-\lambda_{n}}, \quad m=1, \ldots, M .
$$

Remark 1. In case of the Frobenius manifold $M_{\phi}$ from section 2.1.1 the rotation coefficients $\gamma_{m n}$ and the quadratic Hamiltonians are independent of the primary differential $\phi$, i.e. are the same for all Frobenius structures on the Hurwitz space $\hat{H}_{g, N}\left(k_{1}, \ldots, k_{l}\right)$.

Remark 2. The matrices $A_{k}$ that appear in isomonodromy problem (1) are defined in terms of the $V_{m n}$ by $A_{k}=\left\|a_{m n}^{k}\right\|_{m, n=1, \ldots M}$ where $a_{m n}^{k}=0$ if $m \neq k$ and $a_{k n}^{k}=V_{k n}$ for $n=1, \ldots M$.

Remark 3. The function $\tau_{I}$ is only defined up to an overall multiplicative constant. Similarly the $G$ function is only defined up to an overall additive constant. Such numerical factors (and a multiplicative factor in $J$ ) will be ignored.

2.1.3. Quadratic Hamiltonians and Hurwitz spaces. If a semisimple Frobenius manifold $M$ is constructed by means of a Hurwitz space then the corresponding quadratic Hamiltonians (5) admit an alternative expression first found in [10]. To give this expression we need to introduce the so-called Bergman projective connection on the Riemann surface $\mathcal{L}$ and choose a special system of local parameters on $\mathcal{L}$ connected with the covering $p: \mathcal{L} \rightarrow \mathbb{P}^{1}$ from the space $H_{g, N}\left(k_{1}, \ldots, k_{l}\right)$.

First recall the definition of the Bergman kernel. In the case $g>0$ the Bergman kernel on the Torelli marked Riemann surface $\mathcal{L}$ is defined by $B(P, Q)=$ $d_{P} d_{Q} \log E(P, Q)$, where $E(P, Q)$ is the prime-form on $\mathcal{L}$ (see [5]). On the diagonal $P=Q$ the Bergman kernel is singular:

$$
B(x(P), x(Q))=\left(\frac{1}{(x(P)-x(Q))^{2}}+H(x(P), x(Q)) d x(P) d x(Q),\right.
$$

where

$$
H(x(P), x(Q))=\frac{1}{6} S_{B}(x(P))+o(1)
$$

as $P \rightarrow Q$. Here $x(P)$ is a local coordinate of a point $P \in \mathcal{L}, S_{B}$ is the Bergman projective connection (see, e.g., [5],[15]). Being a projective conection, $S_{B}$ depends on the choice of local parameter. If $g=0$ and $z: \mathcal{L} \rightarrow \mathbb{P}^{1}$ is a biholomorphic map then the Bergman kernel is defined by

$$
B(z(P), z(Q))=\frac{d z(P) d z(Q)}{(z(P)-z(Q))^{2}} .
$$

(In particular $S_{B}(z) \equiv 0$ in the local parameter $z$.) 
Let $\lambda$ the standard local parameter on $\mathbb{C} P^{1} \backslash\{\infty\}$. This local parameter gives rise to a local parameter $\lambda(P)\left(P \in \mathcal{L}, \lambda(P)=p(P)\right.$, where $p: \mathcal{L} \rightarrow \mathbb{P}^{1}$ is the covering map) on the Riemann surface $\mathcal{L}$ which is suitable everywhere except in neighbourhoods of the ramification points $P_{1}, \ldots, P_{M}$ and at the infinities $\infty_{1}, \ldots, \infty_{l}$. In the neighbourhood of the ramification point $P_{m}$ one defines the local parameter $x_{m}(P)$ by the formula $x_{m}(P)=\sqrt{\lambda(P)-\lambda_{m}}$ and in the neighbourhood of the point $\infty_{s}$ one defines the local parameter $\zeta_{s}(P)$ by the formula $\zeta_{s}(P)=(\lambda(P))^{-1 / k_{s}}$.

The following theorem, proved in [10], relates the quadratic Hamiltonians to the Bergman projective connection:

Theorem 1. Let $M_{\phi}$ be the Frobenius manifold constructed by means of a Hurwitz space $H_{g, N}\left(k_{1}, \ldots, k_{l}\right)$ and a primary differential $\phi$. Let the pair

$$
<\left[p: \mathcal{L} \rightarrow \mathbb{P}^{1}\right] \in H_{g, N}\left(k_{1}, \ldots, k_{l}\right),\left\{a_{\alpha}, b_{\alpha}\right\}_{\alpha=1}^{g}>
$$

(here $\left\{a_{\alpha}, b_{\alpha}\right\}_{\alpha=1}^{g}$ is a canonical basis of cycles on the Riemann surface $\mathcal{L}$ ) be a point of $M_{\phi}$. The quadratic Hamiltonians $H_{m}$ are connected with the Bergman projective connection on the Riemann surface $\mathcal{L}$ as follows

$$
H_{m}=\left.\frac{1}{24} S_{B}\left(x_{m}\right)\right|_{x_{m}=0} ; m=1, \ldots, M .
$$

Thus, the isomonodromic tau-function of the Hurwitz related Frobenius manifold $M_{\phi}$ is subject to the system of equations

$$
\frac{\partial \log \tau_{I}}{\partial \lambda_{m}}=\left.\frac{1}{24} S_{B}\left(x_{m}\right)\right|_{x_{m}=0} ; m=1, \ldots, M .
$$

In the next section we integrate this system explicitly for spaces of branched coverings of genus zero and one.

\subsection{Calculation of $\tau_{I}$.}

2.2.1. Rauch variational formula. Let $p: \mathcal{L} \rightarrow \mathbb{P}^{1}$ be an $N$-fold covering (or more precisely, a representative of the class of equivalent coverings). Moving the critical values $\lambda_{1}, \ldots, \lambda_{M}$ of the map $p$, we deform the covering $\mathcal{L}$. The behaviour of (normalized) holomorphic differentials on $\mathcal{L}$ under such a deformation is described by the classical Rauch variational formula (see, e.g., [9] for the proof of a more general result) which can be written as follows.

Let $g \geq 1$ and let $v$ be a holomorphic differential on $\mathcal{L}$ with fixed $a$-periods. (Recall that we are considering deformations of our covering; this means, in particular, that $v$ is a family of holomorphic differentials on Torelli marked Riemann surfaces.) Then

$$
\frac{\partial v}{\partial \lambda_{k}}(y)=\left.\frac{1}{2} B\left(y, x_{k}\right) v\left(x_{k}\right)\left(d x_{k}\right)^{-2}\right|_{x_{k}=0} .
$$

In the $g=0$ case $\mathcal{L}$ is a rational surface and there exists a unique biholomorphic map $U: \mathcal{L} \rightarrow \mathbb{P}^{1}$ such that $U(P)=[\lambda(P)]^{1 / k_{1}}+o(1)$ as $P \rightarrow \infty_{1}$. (This 
asymptotic condition replaces fixing the $a$-periods of the differential in $g \geq 1$ case.) Then

$$
\frac{\partial\{d U\}}{\partial \lambda_{k}}(y)=\left.\frac{1}{2} B\left(y, x_{k}\right) d U\left(x_{k}\right)\left(d x_{k}\right)^{-2}\right|_{x_{k}=0},
$$

where $B\left(z_{1}, z_{2}\right)=\frac{d z_{1} d z_{2}}{\left(z_{1}-z_{2}\right)^{2}}, z_{1}, z_{2} \in \mathbb{P}^{1}$ is the Bergman kernel on the Riemann sphere. Let

$$
\begin{aligned}
b\left(P_{k}, P_{l}\right) & =\left.\left[B\left(x_{l}, x_{k}\right) d x_{l}^{-1} d x_{k}^{-1}\right]\right|_{x_{k}=0, x_{l}=0}, \quad k \neq l, \\
b\left(P_{l}, \infty_{s}\right) & =\left.\left[B\left(x_{l}, \zeta_{s}\right) d x_{l}^{-1} d \zeta_{s}^{-1}\right]\right|_{x_{l}=0, \zeta_{s}=0} .
\end{aligned}
$$

The following lemma relates these functions to various derivatives. Ultimately these will be used to integrate the system (9).

Lemma 1. Let $d z=f\left(x_{k}\right) d x_{k}$ be a normalized holomorphic differential on the elliptic surface $\mathcal{L}$ written in the local parameter near the point $P_{k}$ and let

$$
f\left(x_{k}\right)=f_{k}+f_{k, 1} x_{k}+f_{k, 2} x_{k}^{2}+O\left(x_{k}^{3}\right)
$$

as $x_{k} \rightarrow 0$. The function $h\left(\zeta_{s}\right)$ is defined in a neighborhood of the point $\infty_{s}$ by $d z=h\left(\zeta_{s}\right) d \zeta_{s}$ and let $h_{s}=\left.h\left(\zeta_{s}\right)\right|_{\zeta_{s}=0}$

With these definitions

$$
\begin{aligned}
\frac{\partial f_{l}}{\partial \lambda_{k}} & =\frac{1}{2} b\left(P_{k}, P_{l}\right) f_{k}, \quad k \neq l, \\
\frac{\partial h_{s}}{\partial \lambda_{k}} & =\frac{1}{2} b\left(P_{k}, \infty_{s}\right) f_{k},
\end{aligned}
$$

and

$$
\frac{\partial \log f_{k}}{\partial \lambda_{k}}=\left.\frac{1}{12} S_{B}\left(x_{k}\right)\right|_{x_{k}=0}+\frac{f_{k, 2}}{2 f_{k}} .
$$

In the rational case equations (12), (13) and (14) hold with the function $f\left(x_{k}\right)$ defined by $d U\left(x_{k}\right)=f\left(x_{k}\right) d x_{k}$ and the function $h\left(\zeta_{s}\right)$ defined by $d U\left(\zeta_{s}\right)=h\left(\zeta_{s}\right) d \zeta_{s}$. If $g=0$ the number $h_{s}$ is defined only for $s>1$.

Proof. Equations (12) and (13) immediately follow from the Rauch formula. To prove (14) observe that

$$
f\left(x_{k}\right) d x_{k}=\left(f_{k}+f_{k, 1} x_{k}+f_{k, 2} x_{k}^{2}+O\left(x_{k}^{3}\right)\right) \frac{d \lambda}{2 \sqrt{\lambda-\lambda_{k}}}
$$

and

$$
\frac{\partial\left\{f\left(x_{k}\right) d x_{k}\right\}}{\partial \lambda_{k}}=\left[\frac{\partial f_{k}}{\partial \lambda_{k}}-\frac{f_{k, 2}}{2}\right]+\frac{f_{k}}{2 x_{k}^{2}}+o(1)
$$


as $x_{k} \rightarrow 0$. On the other hand, owing to the Rauch formula and the asymtotics of the Bergman kernel at the diagonal, one has

$$
\frac{\partial\left\{f\left(x_{k}\right) d x_{k}\right\}}{\partial \lambda_{k}}=\frac{f_{k}}{2 x_{k}^{2}}+\frac{f_{k}}{12} S_{B}\left(x_{k}\right)+o(1)
$$

as $x_{k} \rightarrow 0$. Comparing (15) and (16), one obtains (14).

2.2.2. The Main Theorem. Using the notation of Lemma 1 one may now write down expressions for the isomonodromic tau-function for low genus Hurwitz spaces.

Theorem 2. The isomonodromic tau-function corresponding to the Frobenius structures on the space $H_{0, N}\left(k_{1}, \ldots, k_{l}\right)$ is given by the expression

$$
\tau_{I}=\left\{\frac{\prod_{m=1}^{M} f_{m}}{\prod_{s=2}^{l} h_{s}^{k_{s}+1}}\right\}^{\frac{1}{24}} .
$$

The isomonodromic tau-function corresponding to the Frobenius structures on the space $H_{1, N}\left(k_{1}, \ldots, k_{l}\right)$ is given by the expression

$$
\tau_{I}=\frac{1}{\eta(\varsigma)}\left\{\frac{\prod_{m=1}^{M} f_{m}}{\prod_{s=1}^{l} h_{s}^{k_{s}+1}}\right\}^{\frac{1}{24}},
$$

where $\varsigma$ is the modulus of the elliptic surface $\mathcal{L}, \eta$ is the Dedekind eta-function.

2.2.3. Proof of the main theorem. We recall some useful relations from [5]. (In agreement with the paper [6], we use here the normalization condition $\left(\int_{a} v, \int_{b} v\right)=(1, \varsigma)$ for the normalized holomorphic differential $v$ on the elliptic surface $\mathcal{L}$. In [5] the normalization $\left(\int_{a} v, \int_{b} v\right)=(2 \pi i, \varsigma)$ was used and this explains why the formulae here differ slightly from those in [5].)

Let $\mathcal{L}$ be a marked elliptic surface and let the $b$-period of the normalized differential $v$ be $\varsigma$. Introduce the function $\tilde{\eta}$ by the equation

$$
\tilde{\eta}(\varsigma)=\frac{d}{d \varsigma} \log \eta(\varsigma)
$$

where $\eta$ is the Dedekind eta-function. Owing to the heat equation for thetafunctions we have

$$
\tilde{\eta}(\varsigma)=\frac{1}{12 \pi i} \frac{\theta^{\prime \prime \prime}\left[\begin{array}{c}
\frac{1}{2} \\
\frac{1}{2}
\end{array}\right](0 \mid \varsigma)}{\theta^{\prime}\left[\begin{array}{l}
\frac{1}{2} \\
\frac{1}{2}
\end{array}\right](0 \mid \varsigma)} .
$$

The Bergman kernel of the elliptic surface $\mathcal{L}$ is given by

$$
B(x, y)=\left[\wp\left(\int_{x}^{y} v\right)-4 \pi i \tilde{\eta}(\varsigma)\right] v(x) v(y),
$$


where $\wp$ is the Weierstrass $\wp$-function. Let $z$ be the coordinate on the universal covering $\mathbb{C}$ of the elliptic surface $\mathcal{L}$. For the invariant Wirtinger projective connection $S_{W}$ (see [5]) we have

$$
S_{W}(x)=S_{B}(x)+24 \pi i \tilde{\eta} v^{2}(x)=\{z, x\},
$$

where $\{z, x\}$ is the Schwarzian derivative (see also [15], the last example in $\S 1.3)$. Note that for a rational surface $\mathcal{L}$ the Bergman projective connection coincides with the Wirtinger invariant projective connection and is nothing but the Schwarzian derivative of the map $U$ :

$$
S_{W}(x)=S_{B}(x)=\{U(x), x\} .
$$

\section{Proposition 1.}

- Let $g=0$. Define the function $\mathbf{T}_{\mathbf{0}}\left(\lambda_{1}, \ldots, \lambda_{M}\right)$ by

$$
\mathbf{T}_{\mathbf{0}}\left(\lambda_{1}, \ldots, \lambda_{M}\right)=\log \left\{\frac{\prod_{m=1}^{M} f_{m}}{\prod_{s=2}^{l} h_{s}^{k_{s}+1}}\right\} .
$$

Then for any $k=1, \ldots, M$

$$
\frac{\partial \mathbf{T}_{\mathbf{0}}}{\partial \lambda_{k}}=\left.S_{W}\left(x_{k}\right)\right|_{x_{k}=0}=\left.S_{B}\left(x_{k}\right)\right|_{x_{k}=0}=\left.\left\{U\left(x_{k}\right), x_{k}\right\}\right|_{x_{k}=0} .
$$

- Let $g=1$. Define the function $\mathbf{T}_{\mathbf{1}}\left(\lambda_{1}, \ldots, \lambda_{M}\right)$ by

$$
\mathbf{T}_{\mathbf{1}}\left(\lambda_{1}, \ldots, \lambda_{M}\right)=\log \left\{\frac{\prod_{m=1}^{M} f_{m}}{\prod_{s=1}^{l} h_{s}^{k_{s}+1}}\right\} .
$$

Then for any $k=1, \ldots, M$

$$
\frac{\partial \mathbf{T}_{\mathbf{1}}}{\partial \lambda_{k}}=\left.S_{W}\left(x_{k}\right)\right|_{x_{k}=0}=\left.S_{B}\left(x_{k}\right)\right|_{x_{k}=0}+24 \pi i \tilde{\eta} f_{k}^{2}=\left.\left\{z, x_{k}\right\}\right|_{x_{k}=0} .
$$

Proof. Using Lemma 1 and formula (20) one obtains

$$
\begin{aligned}
\partial_{\lambda_{k}} \mathbf{T}_{\mathbf{1}}= & \frac{1}{2} \sum_{m \neq k} \frac{b\left(P_{m}, P_{k}\right) f_{k}}{f_{m}}+\left.\frac{1}{12} S_{B}\left(x_{k}\right)\right|_{x_{k}=0} \\
& +\frac{f_{k, 2}}{2 f_{k}}-\frac{1}{2} \sum_{s=1}^{l}\left(k_{s}+1\right) \frac{b\left(P_{k}, \infty_{s}\right) f_{k}}{h_{s}}, \\
= & \left\{\frac{1}{2} \sum_{m \neq k} \wp\left(\int_{P_{m}}^{P_{k}} v\right)-\frac{1}{2} \sum_{s=1}^{l}\left(k_{s}+1\right) \wp\left(\int_{\infty_{s}}^{P_{k}} v\right)\right\} f_{k}^{2}+\left.\frac{1}{12} S_{B}\left(x_{k}\right)\right|_{x_{k}=0} \\
& +\frac{f_{k, 2}}{2 f_{k}}-2 \pi i(M-1) \tilde{\eta} f_{k}^{2}+2 \pi i \sum_{s=1}^{l}\left(k_{s}+1\right) \tilde{\eta} f_{k}^{2} .
\end{aligned}
$$


Using (21), together with the Riemann-Hurwitz formula $M=l+\sum_{s=1}^{l} k_{s}$, this simplifies to

$$
\partial_{\lambda_{k}} \mathbf{T}_{\mathbf{1}}=\frac{1}{2}\left\{\sum_{m \neq k} \wp\left(\int_{P_{m}}^{P_{k}} v\right)-\sum_{s=1}^{l}\left(k_{s}+1\right) \wp\left(\int_{\infty_{s}}^{P_{k}} v\right)\right\} f_{k}^{2}+\frac{f_{k, 2}}{2 f_{k}}+\left.\frac{1}{12} S_{W}\left(x_{k}\right)\right|_{x_{k}=0} .
$$

The analogous formula in the $g=0$ case is

$$
\partial_{\lambda_{k}} \mathbf{T}_{\mathbf{0}}=\frac{1}{2}\left\{\sum_{m \neq k} \frac{1}{\left(z_{k}-z_{m}\right)^{2}}-\sum_{s=2}^{l} \frac{k_{s}+1}{\left(z_{k}-y_{s}\right)^{2}}\right\} f_{k}^{2}+\frac{f_{k, 2}}{2 f_{k}}+\left.\frac{1}{12} S_{W}\left(x_{k}\right)\right|_{x_{k}=0} .
$$

where $z_{m}=U\left(P_{m}\right) ; m=1, \ldots, M$ and $y_{s}=U\left(\infty_{s}\right) ; s=2, \ldots, l$.

In the $g=0$ case let $R: \mathbb{P}^{1} \rightarrow \mathbb{P}^{1}$ be the composition $p \circ U^{-1}$ (we recall that $p: \mathcal{L} \rightarrow \mathbb{P}^{1}$ is the chosen covering from the Hurwitz space). Then $R$ is a rational function and the expression in large braces in (28) coincides with

$$
\left.\left[-\frac{d}{d z}\left(\frac{R^{\prime \prime}(z)}{R^{\prime}(z)}\right)-\frac{1}{\left(z-z_{k}\right)^{2}}\right]\right|_{z=z_{k}}
$$

Similarly, in the $g=1$ case let $\Delta$ be a fundamental parallelogram on the universal covering $\mathbb{C}$ of the elliptic surface $\mathcal{L}$ and let $\mathcal{U}^{-1}: \Delta \rightarrow \mathcal{L}$ be the uniformization map. Let $\mathcal{R}=p \circ \mathcal{U}^{-1}$. Then $\mathcal{R}$ is an elliptic function and the expression in large braces in $(27)$ coincides with

$$
\left.\left[-\frac{d}{d z}\left(\frac{\mathcal{R}^{\prime \prime}(z)}{\mathcal{R}^{\prime}(z)}\right)-\wp\left(\int_{z_{k}}^{z} d z\right)\right]\right|_{z=z_{k}} .
$$

Here we are denoting the standard local parameters on $\mathbb{P}^{1}$ and $\Delta$ by $z$ and, in the last equation, $z_{k} \in \Delta, \mathcal{U}^{-1}\left(z_{k}\right)=P_{k}$.

From now on the proofs of (24) and (26) coincide verbatim and only the details of the rational case will be presented. Let $x=z-z_{k}$ and define $\alpha, \beta$ and $\gamma$ by the condition that

$$
R^{\prime}(z)=\alpha x+\beta x^{2}+\gamma x^{3}+O\left(x^{4}\right)
$$

as $z \rightarrow z_{k}$. It then follows that

$$
\left(\frac{d z}{d x_{k}}\right)^{2}=\frac{4\left(R(z)-R\left(z_{k}\right)\right)}{\left[R^{\prime}(z)\right]^{2}}=\frac{2}{\alpha}-\frac{8}{3} \frac{\beta}{\alpha^{2}} x+\left(\frac{10}{3} \frac{\beta^{2}}{\alpha^{3}}-3 \frac{\gamma}{\alpha^{2}}\right) x^{2}+O\left(x^{3}\right)
$$

and, in particular, that

$$
f_{k}^{2}=\frac{2}{\alpha}
$$

Similarly,

$$
\frac{f_{k, 2}}{2 f_{k}}=\lim _{x_{k} \rightarrow 0} \frac{z^{\prime \prime}\left(x_{k}\right)-z^{\prime \prime}(0)}{4\left(z\left(x_{k}\right)-z(0)\right)}=\frac{5}{6} \frac{\beta^{2}}{\alpha^{3}}-\frac{3}{4} \frac{\gamma}{\alpha^{2}} .
$$


Using the expansion (31) it is straightforward to show that

$$
\begin{gathered}
\frac{2 \gamma \alpha-\beta^{2}}{\alpha^{2}}=\left.\left[\frac{d}{d z}\left(\frac{R^{\prime \prime}(z)}{R^{\prime}(z)}\right)+\frac{1}{\left(z-z_{k}\right)^{2}}\right]\right|_{z=z_{k}} \\
=\left.\left[\{R(z), z\}+\frac{1}{2}\left(\frac{R^{\prime \prime}(z)}{R^{\prime}(z)}\right)^{2}+\frac{1}{\left(z-z_{k}\right)^{2}}\right]\right|_{z=z_{k}} .
\end{gathered}
$$

On using the transformation property of the Schwarzian derivative, together with equations (33) and (32), one obtains

$$
\begin{aligned}
& \frac{4 \gamma \alpha-2 \beta^{2}}{\alpha^{3}} \\
& =\left.\left[\{R(z), z\}\left(\frac{d z}{d x_{k}}\right)^{2}+\left(\frac{1}{2}\left(\frac{R^{\prime \prime}(z)}{R^{\prime}(z)}\right)^{2}+\frac{1}{\left(z-z_{k}\right)^{2}}\right)\left(\frac{d z}{d x_{k}}\right)^{2}\right]\right|_{z=z_{k}} \\
& =\left.\left[\left\{R(z), x_{k}\right\}-\left\{z, x_{k}\right\}+\left(\frac{1}{2}\left(\frac{R^{\prime \prime}(z)}{R^{\prime}(z)}\right)^{2}+\frac{1}{\left(z-z_{k}\right)^{2}}\right)\left(\frac{d z}{d x_{k}}\right)^{2}\right]\right|_{z=z_{k}} \\
& =\frac{\gamma}{\alpha^{2}}-\left.\left\{z, x_{k}\right\}\right|_{x_{k}=0},
\end{aligned}
$$

so

$$
\left.\left\{z, x_{k}\right\}\right|_{x_{k}=0}=\frac{2 \beta^{2}-3 \alpha \gamma}{\alpha^{3}} .
$$

Finally, substituting into (28) the expressions (35), (34) and (36), we get

$$
\begin{aligned}
\partial_{\lambda_{k}} \mathbf{T}_{\mathbf{0}} & =\frac{1}{12}\left(\frac{2 \beta^{2}-3 \alpha \gamma}{\alpha^{3}}\right)-\frac{3}{4} \frac{\alpha \gamma}{\alpha^{3}}+\frac{5}{6} \frac{\beta^{2}}{\alpha^{3}}+\frac{\beta^{2}-2 \gamma \alpha}{\alpha^{3}} \\
& =\frac{2 \beta^{2}-3 \alpha \gamma}{\alpha^{3}} \\
& =\left.\left\{z, x_{k}\right\}\right|_{x_{k}=0} \cdot \square
\end{aligned}
$$

Theorem 2 immediately follows from equations (9), proposition 1 and the Rauch formula

$$
\frac{\partial \varsigma}{\partial \lambda_{k}}=\pi i f_{k}^{2}
$$

a proof of which can be found in [9].

\section{The space of branched coverings of genus 0}

As commented on earlier, Hurwitz spaces $H_{0, N}\left(k_{1} \ldots, k_{l}\right)$ are particularly simple: firstly the spaces $\hat{H}_{g, N}$ and $H_{g, N}$ coincide and secondly the maps $p$ : 
$\mathbb{P}^{1} \rightarrow \mathbb{P}^{1}$ are just rational functions. Such a rational map may, in a suitable coordinate system, be taken to $b^{3}$

$$
p(z)=z^{k_{1}}+\sum_{r=0}^{k_{1}-2} a_{r} z^{r}-\sum_{i=2}^{l} \sum_{\alpha^{i}=1}^{k_{i}} \frac{c_{\left(i, \alpha^{i}\right)}}{\left(z-b^{i}\right)^{\alpha^{i}}},
$$

and in order for $p$ to be well defined the poles $b^{i}$ must be distinct and the coefficients $c_{\left(i, k_{i}\right)}$ at the end of the various Laurent tails must be non-zero. Thus

$$
H_{0, N}\left(k_{1}, \ldots, k_{l}\right) \cong \mathbb{C}^{M} \backslash\left\{b^{i}-b^{j}=0, i \neq j\right\} \cup\left\{c_{\left(i, k_{i}\right)}=0\right\} .
$$

The main additional object is a choice of a primary differential. Here this may be taken to be $\phi=d z$. With this the flat coordinates

$$
\left\{t^{\left(i, \alpha^{i}\right)}: i=1, \ldots, l, \alpha^{i}=2, \ldots, k_{i}\right\} \cup\left\{p^{i}, q^{i}: i=2, \ldots, l\right\}
$$

are given by Theorem 5.1 of [1]. For future notational convenience we define $t_{i}=t^{\left(i, k_{i}\right)}$. With the above formulae for $p$ and $\phi$ one may evaluate these formulae, obtaining, in particular

$$
\left.\begin{array}{rl}
p^{i} & =b^{i} \\
t_{i} & \left.=k_{i} c_{\left(i, k_{i}\right)}\right)^{1 / k_{i}}
\end{array}\right\} \quad i=2, \ldots, l,
$$

so

$$
H_{0, N}\left(k_{1}, \ldots, k_{l}\right) \cong \mathbb{C}^{M} \backslash \mathcal{S}_{1} \cup \mathcal{S}_{2}
$$

where

$$
\begin{aligned}
& \mathcal{S}_{1} \cong\left\{p^{i}-p^{j}=0, i \neq j\right\}, \\
& \mathcal{S}_{2} \cong\left\{t_{i}=0, i=2 \ldots, l\right\} .
\end{aligned}
$$

3.1. The $G$-function in flat coordinates. Theorem 2 together with (2) enables the $G$-function to be calculated explicitly in flat-coordinates. Owing to some cancellations it turns out to be easier to calculate the $G$-function than the $\tau_{I^{-}}$function. A separate calculation of the Jacobian $J$ then enables the $\tau_{I^{-}}$ function to be found.

Theorem 3. The G-function for the Hurwitz space $H_{0, N}\left(k_{1}, \ldots, k_{l}\right)$ is given by

$$
G=-\frac{1}{24} \sum_{i=2}^{l}\left(k_{i}+1\right) \log t_{i} .
$$

The scaling anomaly $\gamma=\mathcal{L}_{E} G$ is given by

$$
\gamma=-\frac{1}{24}\left(l-2+\sum_{i=1}^{l} k_{i}^{-1}+k_{1}^{-1} M\right) .
$$

\footnotetext{
${ }^{3}$ In this formula for $p$, and in various formulae to come, slightly different forms are required if $k_{1}=1$, or in some, if $k_{i}=1$. The final results, though, are not sensitive to this, so only the generic calculations will be presented here.
} 
Proof The proof is really only an extension of the examples in [10] coupled with the flat-coordinate calculation given above. The formula for the $G$-function is:

$$
G=\frac{1}{24} \log \left\{\frac{\left.\prod_{r=1}^{M} \frac{d z}{d x_{r}}\right|_{x_{r}=0}}{\prod_{s=2}^{l}\left(\frac{d z}{d \xi_{s}}\right)^{k_{s}+1}\left(\prod_{r=1}^{M} \underset{P_{r}}{\operatorname{res}} \frac{\phi^{2}}{d \lambda}\right)^{\frac{1}{2}}}\right\} .
$$

Since

$$
p=\frac{c_{\left(i, k_{i}\right)}+O\left(z-b^{i}\right)}{\left(z-b^{i}\right)^{k_{i}}}
$$

and $p=\xi^{-k_{i}}$ around $b^{i}$ one may obtain

$$
\left.\frac{d z}{d \xi_{i}}\right|_{\xi_{i}=0}=c_{\left(i, k_{i}\right)}^{k^{-1}} .
$$

Also

$$
\underset{P_{m}}{\operatorname{res}} \frac{\phi^{2}}{d \lambda}=\left.\frac{1}{2^{M}} \frac{d z}{d x_{m}}\right|_{x_{m}=0},
$$

so the terms which are hard to compute in the numerator and denominator cancel, leaving only a products which have already been evaluated. Hence

$$
G=-\frac{1}{24} \sum_{m=2}^{l}\left(\frac{k_{i}+1}{k_{i}}\right) \log c_{\left(i, k_{i}\right)} .
$$

Expressing this in flat coordinates yields the final result. Note that $G$ is nonsingular at all points in $H_{0, N}\left(k_{1} \ldots k_{l}\right)$; the singularities occurring only on the component $\mathcal{S}_{2}$ of the boundary of the Hurwitz space.

The Hurwitz space $H_{0, N}(N)$ is isomorphic to the orbit space $\mathbb{C}^{N-1} / A_{N-1}$, and in this case one obtains $G=0$. The Hurwitz space $H_{0, N}(k, N-k)$ is isomorphic to the orbit space $\mathbb{C}^{N} / \widetilde{W}^{(k)}\left(A_{N-1}\right)$, where $\widetilde{W}^{(k)}\left(A_{N-1}\right)$ is an extended affine Weyl group [3], and in this case one obtains the formulae derived in [14], though in a slightly different form due to a different primary differential having being used there. Such a change in the primary differential induces a symmetry in the corresponding Frobenius manifolds and this in turn induces a simple transformation for the $G$-function (see Lemma 9 in [14]).

3.2. The $\tau_{I}$-function in flat coordinates. For $p(z)$ given above, its derivative may be written uniquely as

$$
p^{\prime}(z)=\frac{f(z)}{g(z)}
$$


where

$$
g(z)=\prod_{i=2}^{l}\left(z-b^{i}\right)^{k_{i}+1} .
$$

The canonical coordinates are given in terms of the zeroes of $p^{\prime}$,

$$
\left.\begin{array}{rl}
p^{\prime}\left(\alpha^{i}\right) & =0 \\
p\left(\alpha^{i}\right) & =\lambda^{i}
\end{array}\right\} \quad i=1, \ldots M .
$$

By calculating the resultant of $f$ and $g$ one can see that they have no common roots on $H_{0, N}\left(k_{1}, \ldots, k_{l}\right)$ and so the zeroes of $p^{\prime}$ are precisely the roots of the polynomial $f$.

Lemma 2. The resultant $\mathcal{R}(f, g)$ of the polynomials $f$ and $g$ defined by (38) is given by

$$
\mathcal{R}(f, g)=\left\{\prod_{i \neq j}\left(p^{i}-p^{j}\right)^{\left(k_{i}+1\right)\left(k_{j}+1\right)}\right\}\left\{\prod_{i=1}^{m} t_{i}^{k_{i}\left(k_{i}+1\right)}\right\}
$$

where $\left\{p^{i}, t_{i}\right\}$ are flat coordinates for the metric $\xi$.

Proof Writing $f$ as

$$
f(z)=k_{1} \prod_{i=1}^{M}\left(z-\alpha^{i}\right)
$$

gives

$$
\mathcal{R}(f, g)=\prod_{i=1}^{M} \prod_{j=2}^{l}\left(\alpha^{i}-p^{i}\right)^{k_{i}+1} .
$$

But from differentiating the expansion of $p$,

$$
c_{\left(i, k_{i}\right)}=\frac{\prod_{j=1}^{M}\left(p^{i}-\alpha^{j}\right)}{\prod_{i \neq j}\left(p^{i}-p^{j}\right)^{k_{j}+1}}
$$

Using the expression of $c_{\left(i, k_{i}\right)}$ in terms of flat coordinates completes the proof.

Note that $\mathcal{R}(f, g)$ is a non-zero function on $H_{0, N}\left(k_{1} \ldots k_{l}\right)$ : it vanishes precisely on boundary $\mathcal{S}_{1} \cup \mathcal{S}_{2}$. The values of $p^{\prime \prime}\left(\alpha^{i}\right)$, or rather the product of these values, are now easily calculated using basic properties of the resultant:

$$
p^{\prime \prime}\left(\alpha^{i}\right)=\frac{f^{\prime}\left(\alpha^{i}\right)}{g\left(\alpha^{i}\right)}
$$

and hence

$$
\prod_{i=1}^{M} p^{\prime \prime}\left(\alpha^{i}\right)=\frac{\prod_{i=1}^{M} f^{\prime}\left(\alpha^{i}\right)}{\prod_{i=1}^{M} g\left(\alpha^{i}\right)}=\frac{\mathcal{R}\left(f, f^{\prime}\right)}{\mathcal{R}(f, g)}
$$


This then gives the Jacobian $J$. Finally, putting equation (2), Theorem 2 and Lemma 3 together gives:

Theorem 4. The isomonodromic $\tau$-function for the space $H_{0, N}\left(k_{1}, \ldots, k_{l}\right)$ is given by the formula

$$
\tau_{I}^{-48}=\frac{\mathcal{R}\left(f, f^{\prime}\right)}{\left\{\prod_{i \neq j}\left(p^{i}-p^{j}\right)^{\left(k_{i}+1\right)\left(k_{j}+1\right)}\right\}\left\{\prod_{i=2}^{l} t_{i}^{\left(k_{i}+1\right)\left(k_{i}-2\right)}\right\}} .
$$

This formula shows a number of different things. Firstly, $\tau_{I}^{-48}$ is the ratio of quasi-homogeneous polynomials. Moreover the denominator is non-zero at all points in $H_{0, N}\left(k_{1} \ldots k_{l}\right)$, vanishing only on the boundary $\mathcal{S}_{1} \cup \mathcal{S}_{2}$. Hence the zero locus

$$
\left\{\mathbf{t} \in \mathcal{M}: \tau_{I}^{-48}=0\right\}=\left\{\mathcal{R}\left(f, f^{\prime}\right)=0\right\}=\mathcal{K}
$$

that is, the classical caustic where two or more of the $\alpha^{i}$ are coincide. Thus $\tau_{I}^{-48}$ is non-zero on $\mathcal{M} \backslash \mathcal{K}$ and zero only on $\mathcal{K}$. Such a caustic is stratified by the multiplicities of the roots of $f(z)=0$.

The quasihomogeneous polynomial $\mathcal{R}\left(f, f^{\prime}\right)$ is not irreducible:

Proposition 2. The resulatnt $\mathcal{R}\left(f, f^{\prime}\right)$ factorizes:

$$
\mathcal{R}\left(f, f^{\prime}\right)=\left\{\prod_{\left\{i: k_{i}=1\right\}} t_{i}\right\} \cdot\left\{\prod_{r<s}\left(p^{r}-p^{s}\right)^{m_{r s}}\right\} \cdot \widetilde{\mathcal{R}}\left(f, f^{\prime}\right),
$$

where $\widetilde{\mathcal{R}}\left(f, f^{\prime}\right)$ is a quasihomogeneous polynomial.

Proof To derive this we use the property of the resultant that there exists functions $\alpha(z)$ and $\beta(z)$ (which here will be quasihomogeneous polynomials) such that

$$
\mathcal{R}\left(f, f^{\prime}\right)=\alpha(z) f(z)+\beta(z) f^{\prime}(z) .
$$

The function $f$ is explicitly

$$
f(z)=\prod_{i=2}^{l}\left(z-b^{i}\right)^{k_{i}+1} \cdot\left\{k_{1} z^{k_{1}-1}+\ldots+\sum_{i=2}^{l} \sum_{\alpha^{i}=1}^{k_{i}} \frac{\alpha^{i} c_{\left(i, \alpha^{i}\right)}}{\left(z-b^{i}\right)^{\alpha^{i}+1}}\right\}
$$

and this may be used to calculate $f\left(b^{i}\right)$ and $f^{\prime}\left(b^{i}\right)$. For example

$$
f\left(b^{i}\right)=t_{i} \prod_{r \neq i}\left(b^{i}-b^{r}\right)^{k_{r}+1}
$$

and a similar calculation show that $f^{\prime}\left(b^{i}\right)$ has a factor $t_{i}$ if and only if $k_{i}=1$. Hence $\mathcal{R}$ has a factor of $t_{i}$ if and only if $k_{i}=1$. A similar argument gives the second factor in the above formula with $m_{r s}=\left(k_{r}+k_{s}\right)$. 
The $m_{r s}$ factor is not optimal, as may be shown by calculating certain examples. It seem likely, based on the explicit calculation of low dimensional examples, that with an optimal factor, the residual polynomial $\widetilde{\mathcal{R}}\left(f, f^{\prime}\right)$ is irreducible.

\section{The space of branched coverings of genus 1}

Elliptic functions may easily be manipulated in the same way as rational functions. Here the definitions and properties of these and related functions will follow [16]. Any elliptic function may be written as

$$
p(z)=a+\sum_{i=1}^{l} \sum_{\alpha^{i}=1}^{k_{i}} c_{\left(i, \alpha_{i}\right)} \zeta^{\left(\alpha^{i}-1\right)}\left(z-b_{i}\right)
$$

with the local expansion around the pole $z=b_{i}$,

$$
\frac{c_{i, 1}}{z-b_{i}}-\frac{c_{(i, 2)}}{\left(z-b_{i}\right)^{2}}+\ldots+\frac{\left(k_{i}-1\right) !(-1)^{k_{i}-1} c_{\left(i, k_{i}\right)}}{\left(z-b_{i}\right)^{k_{i}}} .
$$

In order for this to be well-defined, the poles must be distinct with well-defined orders, so singular spaces $\mathcal{S}_{1}$ and $\mathcal{S}_{2}$ may be defined as above. Such an elliptic function admits an alternative representation in terms of products of $\sigma$-functions,

$$
p(z)=a_{0} \frac{\prod_{i=1}^{N} \sigma\left(z-a_{i}\right)}{\prod_{j=1}^{l} \sigma\left(z-b_{j}\right)^{k_{j}}}
$$

with $\sum_{i=1}^{N} a_{i}=\sum_{j=1}^{l} k_{j} b_{j}$. To proceed further one requires an elliptic version of the resultant of two polynomials. This may be defined as follows. Let

$$
F(z)=a_{0} \prod_{i=1}^{M} \sigma\left(z-a_{i}\right), \quad G(z)=b_{0} \prod_{j=1}^{N} \sigma\left(z-b_{j}\right)
$$

and define $\mathcal{R}(F, G)=a_{0}^{N} \prod_{i=1}^{M} G\left(a_{i}\right)$. It follows immediately from this that

$$
\mathcal{R}(F, G)=a_{0}^{N} b_{0}^{M} \prod_{i, j} \sigma\left(a_{i}-b_{j}\right)=(-1)^{M N} \prod_{j=1}^{N} F\left(b_{j}\right)=(-1)^{M N} \mathcal{R}(G, F) .
$$

In particular, $\mathcal{R}(F, G)=0$ if and only if $F$ and $G$ have, modulo the lattice, a common zero, this following from properties of the $\sigma$-function. For arbitrary $F$ and $G$ of this form, the elliptic resultant $\mathcal{R}(F, G)$ does not transform invariantly under the lattice transformations $a_{i} \mapsto a_{i}+2 m \omega_{1}+2 n \omega_{2}, b_{i} \mapsto b_{i}+2 m \omega_{1}+2 n \omega_{2}$. However, if $F / G$ is an elliptic function then the elliptic resultant transforms as

$$
\mathcal{R}(F, G) \mapsto c_{L}(m, n) \mathcal{R}(F, G)
$$

where $c_{L}(m, n)$ is a non-zero function, depending on the periods.

The $G$-function and $\tau_{I}$-function for the space $H_{1, N}\left(k_{1}, \ldots, k_{l}\right)$ may now be calculated in manner entirely analogous to Theorem 3 and 4 . 
Theorem 5. The $G$-function for the Hurwitz space $H_{1, N}\left(k_{1}, \ldots, k_{l}\right)$ is given by

$$
G=-\log \eta\left(t_{0}\right)-\frac{1}{24} \sum_{i=2}^{l}\left(k_{i}+1\right) \log t_{i} .
$$

The scaling anomaly $\gamma=\mathcal{L}_{E} G$ is given by

$$
\gamma=-\frac{1}{24}\left(l+\sum_{i=1}^{l} k_{i}^{-1}+k_{1}^{-1} M\right) .
$$

Proof The proof is, again, really only an extension of the example in [10], so

$$
\left(\prod_{m=1}^{M} \operatorname{Res}_{P_{m}} \frac{\phi^{2}}{d \lambda}\right)=\mathrm{const}\left(\left.\prod_{m=1}^{M} \frac{\omega\left(x_{m}(P)\right)}{d x_{m}(P)}\right|_{P=P_{m}}\right)^{2}=\left(\prod_{m=1}^{M} f_{m}\right)^{2}
$$

and the flat coordinate calculations

$$
t_{0}=\oint_{b} \omega=\varsigma
$$

and

$$
\begin{aligned}
t_{i} & =t^{\left(i, \alpha_{i}\right)}=\operatorname{Res}_{z=0}\left(z[\lambda(z)]^{-\frac{k_{i}-1}{k_{i}}} d \lambda(z)\right) \\
& =\operatorname{Res}_{\zeta_{i}=0}\left(z\left(\zeta_{i}\right) \frac{d \zeta_{i}}{\zeta_{i}^{2}}\right)=\left.z^{\prime}\left(\zeta_{i}\right)\right|_{\zeta_{i}=0}=h_{i} \\
& =\operatorname{const} c_{\left(i, k_{i}\right)}{ }^{1 / k_{i}} \cdot \square
\end{aligned}
$$

In particular, for the space $H_{1, N}(N)$ one obtain the $G$-function conjectured in [14] and proved in [10] (N.B. a log-term went missing in [10] and a different normalization was used [14] which accounts for the different numerical factor in front of the first term in the $G$-function above).

Since $p^{\prime}(z)$ is also an elliptic function it may be written as $p^{\prime}=f / g$ where $f$ and $g$ are products of $\sigma$-functions. The proof of Theorem 4 goes through verbatim with the elliptic resultant introduced above. In particular, the function $\mathcal{R}(f, g)$ can be calculated in exactly the same way as above.

Theorem 6. The isomonodromic $\tau$-function for the space $H_{1, N}\left(k_{1}, \ldots, k_{l}\right)$ is given by the formula

$$
\tau_{I}^{-48}=\frac{\eta\left(t_{0}\right)^{48} \kappa}{\left\{\prod_{i \neq j}\left(b^{i}-b^{j}\right)^{\left(k_{i}+1\right)\left(k_{j}+1\right)}\right\}\left\{\prod_{i=1}^{l} t_{i}^{\left(k_{i}+1\right)\left(k_{i}-2\right)}\right\}}
$$

where

$$
\kappa=\prod_{r \neq s} \sigma\left(\alpha_{r}-\alpha_{s}\right)
$$

and $\alpha_{i}, i=1, \ldots, M$, are the critical points of the map $p$. 
The submanifold $\kappa^{-1}(0)$ corresponds to the caustic $\mathcal{K}$ where the multiplication is non-semisimple.

Example The Hurwitz space $H_{1,2}(2)$ was studied in detail in [1], appendix C. Detailed calculations give

$$
\tau_{I}^{-48}=t_{1}^{12} \hat{\Delta}^{3}\left(t_{0}\right)
$$

where $\hat{\Delta}\left(\omega^{\prime} / \omega\right)=\omega^{12} \Delta\left(\omega, \omega^{\prime}\right)$ and $\Delta$ is the classical discriminant of the underlying elliptic curve with periods $\omega$ and $\omega^{\prime}$, i.e., given an elliptic curve $y^{2}=$ $4 x^{3}-g_{2}\left(\omega, \omega^{\prime}\right) x-g_{3}\left(\omega, \omega^{\prime}\right)$ the discriminant is defined by $\Delta=g_{2}^{3}-27 g_{3}^{2}$. This shows that $\tau_{I}$ is singular where $\Delta=0$, that is, where the underlying elliptic curves develops a singularity. Such points are not strictly speaking in the Hurwitz space - they occur on its boundary.

\section{Comments}

While for any individual Frobenius manifold one may find the corresponding $G$-function by directly solving the defining differential equation, calculating it explicitly for classes of Frobenius manifolds is more problematic. For orbit spaces $\mathbb{C}^{M} / W$ where $W$ is a Coxeter group, the $G$-function was calculated by studying the $F$-manifold structure on the caustics. Such an approach could be applied to other classes of manifolds, such as the Hurwitz spaces in this paper, but this is hampered by the lack of information on the $F$-manifold structure on the caustics. The formulae $(37,39)$ suggest that one has a number of logarithmic caustics $\mathcal{K}_{\log }$ and that the multiplication on them is of the type given in Lemma 2.3 in [14] with $N_{\log }=\left(k_{i}+1\right)$, but to verify this would require much detailed calculation. The methods used here are very specific to Hurwitz spaces. However certain structures appear in both approaches, namely the role of caustics and singular structures (which include the logarithmic caustics introduced in [14]) on the boundary of the Hurwitz spaces. Certainly the results suggest that a detailed study of the caustics and boundaries of Hurwitz spaces should be undertaken. The techniques of this paper could also be applied to the 'real-double' Hurwitz spaces recently introduced by Shramchenko [13].

More generally, both constructions have a considerable global character: the results are all obtained in terms of critical data. This suggests that there might be formulae for the $G$ and $\tau_{I}$ functions in terms of the Saito formalism of Frobenius manifolds.

\section{Acknowledgements}

We would like to thank S. Natanzon and C. Hertling and most particularly D. Korotkin for their various comments and criticisms of this work, and the Max Planck Institute, Bonn, for its hospitality. IABS would like to thank the EPSRC (grant GR/R05093) for financial support. 


\section{References}

[1] B. Dubrovin, Geometry of 2D topological field theories in Integrable Systems and Quantum Groups, (Montecatini Terme, 1993), 120-348, Lecture Notes in Math., 1620, Springer, Berlin, 1996.

[2] B. Dubrovin and Y. Zhang, Bi-Hamiltonian hierarchies in the 2D Topological Field Theory at One-Loop Approximation, Comm. Math. Phys. 198 (1998) 311-361.

[3] _ Frobenius Manifolds and Virasoro Constraints, Selecta Math. (N.S.) 5 (1999) 423-466.

[4] B. Eynard, A. Kokotov, and D. Korotkin, $1 / N^{2}$ correction to free energy in hermitian two-matrix model, Lett. Math. Phys. 71 (2005) 199-207.

[5] J. D. Fay, Theta-functions on Riemann surfaces, Lecture Notes in Mathematics, Vol. 352. Springer-Verlag, Berlin-New York, 1973.

[6] Kernel functions, analytic torsion, and moduli spaces, Mem. Amer. Math. Soc., 96 (1992).

[7] A. B. Givental, Elliptic Gromov-Witten invariants and the generalized mirror conjecture (English summary), Integrable systems and algebraic geometry (Kobe/Kyoto, 1997), 107155, World Sci. Publishing, River Edge, NJ, 1998.

[8] C. Hertling, Frobenius manifolds and moduli spaces for singularities, Cambridge Tracts in Mathematics 151, Cambridge University Press, 2002.

[9] A. Kokotov, D. Korotkin, Tau-functions on Hurwitz spaces, Math. Phys. Anal. Geom. 7 (2004) 47-96.

[10] On G-function of Frobenius manifolds related to Hurwitz spaces, Int. Math. Res. Not. 2004, N7, 344-360.

[11] D. Korotkin, Matrix Riemann-Hilbert problems related to branched coverings of $\mathbb{C P}^{1}$, Factorization and integrable systems (Faro, 2000), 103-129, Oper. Theory Adv. Appl., 141, Birkhuser, Basel, 2003.

[12] V. Shramchenko, private communication.

[13] _ "Real doubles" of Hurwitz Frobenius manifolds, Comm. Math. Phys. 256 (2005) $635-680$

[14] I. A. B. Strachan, Symmetries and solutions of Getzler's equation for Coxeter and extended affine Weyl Frobenius manifolds, Int. Math. Res. Not. 2003, no. 19, 1035-1051.

[15] A. N. Tyurin, Periods of quadratic differentials (Russian), Uspekhi Mat. Nauk 33 , no. 6(204), 149-195 (1978). English translation: Russian Math. Surveys 33 (1978), no. 6, 169-221.

[16] E. T. Whittaker and G. N. Watson, A course of modern analysis. Reprint of the fourth (1927) edition. Cambridge Mathematical Library. Cambridge University Press, Cambridge, 1996.

[17] P. Zograf, L. Takhtajan, On the Liouville equation, accessory parameters and the geometry of Teichmüller space for Riemann surfaces of genus 0, Math. USSR-Sb. 60 (1988) $143-161$.

Department of Mathematics and Statistics, Concordia University, 7141 Sherbrooke West, Montreal H4B 1R6, Quebec, Canada

E-mail address: alexey@mathstat.concordia.ca

Department of Mathematics, University of Glasgow, Glasgow G12 8QW, U.K.

E-mail address: i.strachan@maths.gla.ac.uk 